\title{
Reconstructing individual shape histories of fish otoliths: A new image- based tool for otolith growth analysis and modeling
}

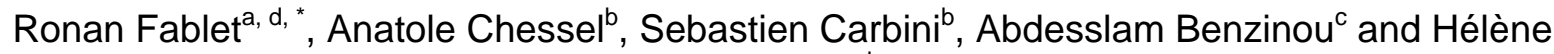 \\ de Pontual ${ }^{b}$
}

\author{
a Institut Telecom/Telecom Bretagne/UMR 3192 LabSTICC, CS 83818, Technopôle Brest-Iroise, 29238 Brest \\ Cedex 3, France \\ ${ }^{\mathrm{b}}$ Ifremer/STH, BP 70, 29280 Plouzané, France \\ ${ }^{c}$ ENIB/RESO, Technopôle Brest-Iroise 29280 Plouzane, France \\ d Université européenne de Bretagne, France
}

*: Corresponding author : Fablet R., email address : ronan.fablet@telecom-bretagne.eu

\begin{abstract}
:
In this paper is presented a novel image processing tool for the extraction of geometric information in otolith images. It relies on the reconstruction of individual otolith shape histories from otolith images. Based on the proposed non-parametric level-set representation of otolith shape history, applications to the extraction of growth axes and ring structures in otolith images are first considered. A second category of applications concern the analysis of $2 \mathrm{D}$ otolith growth. The potential of the proposed framework is illustrated on real otolith images for various species (e.g., cod, pollock) and discussed with a particular emphasis on the genericity of the approach and on applications such as otolith shape analysis, multi-proxy otolith analysis, otolith modeling.
\end{abstract}

Keywords: Otolith imaging; Shape dynamics; Growth ring extraction; Growth axis extraction; 2D otolith growth 


\section{Introduction and problem statement}

As they grow according to an accretionary process, fish otoliths can be viewed as a succession of threedimensional concentric layers. The composition of these layers, in terms of physico-chemical characteristics, vary according to endogenous and exogenous factors [Panfili et al., 2002]. The accretionary process depicts a periodic rhythmicity, typically daily and/or seasonal, deposit, such that the observation of these biological structures in an observation plane going through the initial core depict concentric ring patterns, also called growth marks. These characteristics provide the basis for exploiting these structures as biological archives to define environmental proxies (e.g., for instance to reconstruct temperature series) [Hoie et al., 2004], or to reconstruct individual life traits (e.g., individual age and growth information or migration paths) [Fablet et al., 2007]. To further stress the key importance of these biological structures in marine ecology, it can be pointed out that well over one million of fish [Campana and Thorrold, 2001] are analyzed each year for estimating age structures which are among the key data for fish stock assessment.

Following ongoing developments [Alvarez et al., 2007, Fablet, 2006, Fablet et al., 2007] aimed at information extraction and interpretation in fish otolith images, this paper addresses the extraction of geometric otolith characteristics and their application to otolith growth modelling and analysis. Though extensively studied and exploited [Campana and Casselman, 1993, de Pontual and Prouzet, 1988], the analysis of the shape of fish otoliths and other calcified structures has usually been restricted to the analysis of the outline of the otolith in a given observation plane, especially for stock and species discrimination. However, the presence of internal ring structures potentially provides the mean for back-tracking the evolution of the shape of the otolith from the core to the edge. Such an information is of great interest for analyzing, modelling and extracting the main features of the otolith growth. Recently, we have developed a new computational tool aimed at reconstructing the sequence of the successive shapes associated with an accretionary growth process in a given observation plane containing the otolith core [Fablet et al., 2008b]. We benefit from this 
representation of the otolith growth to develop new solutions for information extraction in otolith images.

Experimental results for various species are reported, and, we investigate a quantitative analysis of the 2D otolith growth, more particularly of the dynamics and of the spatial heterogeneity of shape, growth and opacity feature. The genericity of the approach is further discussed as well as its broad interest for applications to otolith shape analysis, multi-proxy otolith analysis and numerical otolith modelling.

\section{Material and methods}

\section{Otolith material}

In this study, the biological material of interest is provided as images of whole otoliths or otolith sections associated with an interpretation of the internal growth structures in terms of age and growth. Otolith sections have been prepared in the transverse plane. We focus on seasonal growth and thick sections are considered. The proposed methodological developments are evaluated for several species (namely, examples of cod (Gadus morhua), hake (Merluccius merluccius), plaice (Pleuronectes platessa), pollock (Pollachius virens, and whiting (Merlangus merlangus) are considered). These species are chosen as they provide a panel of complexity levels in terms of image contrast and ring structures. This choice is also aimed at demonstrating the improvements compared to previous work [Fablet, 2006, Guillaud et al., 2002, Palmer et al., 2005, Traodec et al., 2000] which were mainly limited to the analysis of whole plaice otoliths.

Otolith images have been acquired under a binocular using transmitted or reflected light depending on the species with a 1000x1000 digital camera.

\section{Reconstruction of individual histories of 2D otolith shapes from images}

The core of the proposed computational framework is the reconstruction of the evolution of the 2D otolith shape in a given observation plane from an image. With a view to modelling and representing the 2D otolith 
growth, we adopt a level-set setting of the accretionary growth process. It relies on the definition of a convex potential function $U$ such that the 2D shape $\Gamma_{t}(U)$ of the otolith at time $t$ is given by a level line of $U$, that is to say the set of points $p$ for which the associated potential value $U(p)$ equals $t$ :

$$
\Gamma_{t}(U)=\left\{p \in \mathcal{R}^{2} \text { such that } U(p)=t\right\}
$$

This level-set representation of the accretionary growth of fish otoliths is illustrated (Fig.1). The potential function $U$ is displayed as a 3D surface, and the successive level-lines of $U$, for potential values uniformly sampled, are visualized in the horizontal plane. This level-set setting is of great interest for several reasons:

- It intrinsically conforms to the requirements that the accretionary growth is normal to the shape and that the successive shapes are concentric;

- It is a compact representation of a series of successive shapes, the whole series of shapes being represented by a single mathematical function $U$;

- It is generic as it accounts for elliptic-like shapes, such as the shapes depicted by whole plaice otoliths, as well as more complex non-convex examples such as the shapes of hake or cod otolith sections;

- It is non-parametric. Contrary to the parametric approach proposed in [Alvarez et al., 2007], no assumption is made on the evolution of the shape, such that subsequent analysis is not biased by some parametric a priori which may not be fulfilled in practice.

[Figure 1 about here.]

Our goal is to fit the level-set model $U$ to an otolith image in a given observation plane, such that the successive level-lines of $U$ match the internal rings of the otolith. We further assume that we are provided with additional constraints, referred to as boundary conditions, at least the position of the nucleus of the 
otolith and the edge of the otolith which can be extracted automatically [Cao and Fablet, 2006]. Additional internal rings may also be provided. Fitting model $U$ is then viewed as its interpolation to the whole image domain given known values at the boundary conditions. This interpolation is stated as the minimization of an energy criterion involving two different terms. The first term is a regularity term setting that the successive shapes $\Gamma_{t}(U)$ should be smooth. This term is computed as the sum of the perimeter of all the shapes $\left\{\Gamma_{t}(U)\right\}$. The second term relies on image-based features. Exploiting previous work on the estimation of local image orientations [Chessel et al., 2006], this term states that the normal to shape $\left\{\Gamma_{t}(U)\right\}$ at point $p$ should be orthogonal to the estimated local orientation, denoted by $w(p)$. An example of a map of local image orientations is reported for a pollock otolith section (Fig.2). Formally, the considered energy criterion is given by:

$$
E(U)=(1-\gamma) \int_{t \in[0, T]} \int_{p \in \Gamma_{t}(U)} 1+\gamma \int_{t \in[0, T]} \int_{p \in \Gamma_{t}(U)} \cdot\left\|\left\langle\frac{\nabla U(p)}{|\nabla U(p)|}, \omega(p)\right\rangle\right\|
$$

where $\gamma$ is a weight setting the relative influence of the two terms, $\nabla U(p) /|\nabla U(p)|$ the orientation of shape $\Gamma_{t}(U)$ at point $p$ and $<\nabla U(p) /|\nabla U(p)|, \omega(p)>$ the scalar product evaluating whether the two orientations are orthogonal. We let the reader to [Fablet et al., 2008a, Fablet et al., 2008b] for details on the numerical implementation of the gradient-based minimization of criterion $E$. Cross-validation experiments carried out on synthetic examples have shown that values of $\gamma$ in the range [0.4,0.8] are optimal [Fablet et al., 2008a]. In the reported experiments, $\gamma$ is set to 0.6. Concerning the computational cost, the proposed scheme is implemented as a C code under Linux and runs in about a minute for a 1000x1000 image.

If the otolith growth pattern along a given growth axis is known, the estimated potential function $U$ provides at any pixel $p$ an age estimate. If not, potential function $U$ only provides the successive 2D shapes of the otolith from the core to the edge. In that case, the values of $U$ refer to the actual age up to a contrast change (i.e., a monotonic increasing function). This second situation occurs when dealing with automated otolith imaging for instance for automated fish ageing [Fablet, 2006]. 


\section{Extraction of geometric structures in otolith images}

A fitted potential function $U$ provides the mean for automatically extracting relevant geometric information from an image. We here illustrate this great potential for three different types of information, namely growth shapes, internal ring structures and growth axis. We briefly review the proposed approaches and let the reader refer to [Fablet et al., 2008a] for detailed presentations on the associated algorithms.

A first straightforward by-product is the sequence of 2D growth shapes of the otolith from as level-lines of potential function $U$. More particularly, if function $U$ is time-calibrated, the 2D shape of the otolith can be extracted at any age, i.e. at any precision (yearly, biannual,...) as a level-line pf $U$. Such information is of key interest regarding otolith shape analysis and classification [Campana and Casselman, 1993] when samples from different age groups have to be dealt with as well as when considering the entire shape history may be a discriminant feature.

A second application is the extraction of the opaque and translucent ring curves. It serves for instance as a basis for age and growth estimation [Fablet, 2006, Traodec et al., 2000]. Good performances have been reported for images of whole plaice otoliths [Traodec et al., 2000, Fablet, 2006, Palmer et al., 2005]. However, these otoliths depict very clear ring structures and their growth can be viewed as mainly radial. For more complex images (eg, hake or cod otolith images), the methods proposed in previous work do not succeed in correctly extracting ring structures. Based on the estimated model $U$, we can address these issues. Growth ring structures correspond to image valleys and ridges (together known as creases), which are the relief curves of the landscape obtained when the image intensity is seen as a height map. We then propose to extract ring curves as portions of the level-lines of $U$ depicting high values of a local contrast-based measure. Formally, our method is implemented within a contrario detection framework [Desolneux et al., 2001, Desolneux et al., 2003].

When focusing on temporal signals archived by fish otoliths (eg, growth patterns, migrations, environ- 
mental records,...), the analysis is mainly one-dimensional from the core to the edge of the otoliths. Hence, the extraction and the standardization of the reference growth axis is a crucial step. To our knowledge, no tool has been developed to this end. Growth axis can indeed be viewed as a by-product of the potential function $U$. As the accretionary growth is normal to the surface, growth axis can be defined as paths linking the growth center to the edge such that these paths are normal to the ring structures. A straightforward solution would then be to extract growth axis as integral lines of the orientation field of $U$. This solution is however numerically unstable and a more robust variational setting is proposed. Using a minimal path scheme [Cohen, 2005], growth axis are retrieved as smooth paths from the core to the edge locally as normal as possible to level-lines of $U$.

\section{Quantitative analysis of the 2D otolith growth}

Whereas a huge amount of work has been dedicated to the extraction and the analysis of one-dimensional otolith growth patterns [Campana and Thorrold, 2001, Panfili et al., 2002], the actual quantitative analysis of the 2D growth has, to our knowledge, only been seldom considered. Such an analysis is of great interest to better understand and characterize the relations between otolith features, such as shape, growth and opacity. In previous work, these issues have only been considered from the global characterization, using for instance mean opacity and growth descriptors for each otolith of a given set from which a statistical analysis was carried out [Hussy et al., 2004]. The methodology proposed here intrinsically differs in the amount and the type of information extracted from each otolith section and the characterization of the otolith sections is considered at finer scales. Five local measures of the accretionary growth process are defined at the same resolution as the processed otolith images are introduced: 


\footnotetext{
${ }^{1}$ For a planar curve, the curvature is the inverse of the curvature radius. The curvature radius of a curve at point $p$ is the radius of the circle tangent to the curve and best fitting to the curve. For instance, the curvature radius of the circle is its radius and straight lines have an infinite curvature radius and equivalently a null curvature.
}

- ring curvature (RC): from the empirical observation of otolith sections, it can be noted that the relative growth is generally greater in the regions of the otolith depicting high curvatures ${ }^{1}$. The local curvature is directly computed from level-lines of $U$ (see [Fablet et al., 2008a] for details);

- otolith growth (OG): assuming that the estimated potential function $U$ is time-calibrated (i.e. internal ring structures have been assigned an age), the 2D map of the growth increments is computed as $1 /\|\nabla U(p)\|$ at any point $p$. Such a map is a direct extension of the estimation of the growth increments along a specific growth axis and then permits comparing the series of growth increments along any set of growth axis;

- otolith growth anisotropy (OGA): we also define a measure of the anisotropy of the 2D otolith growth. Our goal is to analyze relative growth variations in different otolith regions as they may reveal properties of the underlying biomineralisation processes, especially the heterogeneity of the endolymph [Allemand et al., 2007]. The anisotropy measure is defined as the local growth increment subtracted by the median growth increment at the associated age [Fablet et al., 2008a];

- otolith opacity (OO): the opacity is a characteristic of the biomineralizaton. It is widely assumed that it reveals the physico-chemical characteristics of the accretionary deposit (e.g, crystallization properties, characteristics of the mineral and organic fractions of the deposit) in relation to fish metabolism and environmental conditions [Hoie et al., 2008, Hussy and Mosegaard, 2004, Hussy et al., 2004]. Let us stress that the images considered in our analysis are acquired under reflected light such that the greater the opacity the greater the image intensity. 
- relative otolith opacity (ROO): we also define a relative opacity feature with a view to investigating local differences in opacity variations. Similarly to the measurement of growth anisotropy, it is computed as the local opacity subtracted by the median opacity at the associated age [Fablet et al., 2008a].

Given these five local measures of the 2D otolith growth, we aim at exploring three issues regarding the otolith formation:

- Can we model the dynamics of the 2D otolith growth?

- What are the relations between the 2D features of the accretion, especially geometric shape features, growth measures and opacity descriptors?

- Are these relations between the 2D features of the accretion constant in space and time?

To proceed to this quantitative analysis, a key feature of the level-set model of the accretionary growth is that it provides a standardized frame. More precisely, it naturally defines a polar-like representation: the growth axis being the radials, i.e. equivalent to an angular reference, and the age being the distance along an axis. Formally, polar maps are interpolated such that point $(\rho, \theta)$ in the polar image refers to the point in the image along the growth axis $\theta$ at a time distance $\rho$ from the otolith center. This polar analysis can be exploited to spatially discriminate specific otolith zones, such as the ventral and dorsal regions which correspond to different angular sectors in the polar images. Similarly, it permits studying the distribution of the otolith growth features in these zones. Besides, the analysis can also be restricted to specific age intervals.

The reported statistical analysis is carried out using standard statistical tools, such as the factor analysis and correlation statistics, from age 0.5 to age 4 for four different zones in the transverse plane: the dorsal, ventral, distal and proximal zones. 


\section{Results}

\section{Reconstruction of individual histories of 2D otolith shape}

The illustration of the different steps of the reconstruction of the otolith shape history is exemplified with an image of a pollock otolith (Fig.2): in addition to the otolith image (top left) are depicted the estimated orientation field $\omega$ (top right), the estimated potential function $U$ (bottom left), with uniformly sampled level-lines projected onto the horizontal plane, and uniformly sampled level-lines superposed to the otolith image (bottom right). It should be stressed that the depicted level-lines are not aimed at corresponding to image ridges or valleys, as they only result from uniformly sampled potential values of the estimated function $U$. Note that the proposed approach can also exploit closed or partial internal rings to further constrain the reconstruction of potential function $U$ [Fablet et al., 2008a].

[Figure 2 about here.]

[Figure 3 about here.]

Results for three other fish species, namely plaice (Pleuronectes platessa), cod (Gadhus morua) and hake (Merluiccius merluccius) are presented (Fig. 3). The best results are obtained for the whole plaice otolith, as it involves the clearer structures. The results reported for the whiting (Gadhus morua) and cod (Merlangius merlangus) otoliths demonstrate that we are also capable of approximately recovering the complex and non-isotropic evolution of such otolith shapes from images depicting lower contrasts.

\section{Geometric information extraction in otolith images}

Besides the illustration of the extraction of the 2D otolith shapes (Fig.3), the application to the automated extraction of ring structure is reported (Fig.4) for three otolith images: an image of a transverse section of a pollock otolith, an image of a transverse section of a cod otolith and an image of a transverse section of hake 
a otolith. In all cases, the proposed approach detects meaningful ring parts. It should be stressed that the reported results do not involve any postprocessing steps for instance for removing the shorter curves. This is viewed as an additional interpretation step which is application-dependent as for instance for automated ageing in [Fablet, 2006]. The comparison to previous work [Fablet, 2006] demonstrates the significant improvement brought by the proposed approach, especially for complex samples such the hake otolith image.

[Figure 4 about here.]

The automated extraction of the growth axis is carried out for several images (Fig.5). For each otolith image, the growth axes from the otolith center to points equally sampled along the edge of the otolith are extracted. As expected, the extraction of the growth axis stresses that the growth is mainly radial for the considered whole plaice otolith, whereas for the three transverse sections, namely pollock, whiting and cod otoliths, the growth can be regarded as radial only in the distal zone of the otolith. The growth axis reconstructed in the ventral and dorsal zones are especially curved. It may also be noted that the extraction of the growth axis tend to enhance the main growth axis along the ventral-dorsal axis.

[Figure 5 about here.]

\section{Quantitative analysis of 2D otolith growth}

The quantitative analysis of the $2 \mathrm{D}$ otolith growth in the transverse plane is carried out for pollock otolith sections. This species is well-suited for such an analysis, as clear yearly opaque and translucent rings are visible on transverse otolith sections [Hoberman and Jensen, 1962]. The reported experiments are based on the analysis of ten otolith sections of individuals belonging to age groups 4 and 5. These individuals were caught in the Northeast Atlantic and sampled in the auction room of Boulogne/Mer. Our analysis is exemplified with the pollock otolith sections depicted in Fig..2 and statistical tests are evaluated for the considered set of otolith sections. 
We first depict the five different local features of the 2D otolith growth, otolith growth anisotropy, ring curvature, otolith opacity and relative otolith opacity, which the analysis is based on (Fig.6). The visual analysis of these two-dimensional maps of the otolith growth stresses that the greatest the curvature, the greatest the growth. The 2D growth map also illustrates that the fast growth zones, especially in the ventral and dorsal areas, are associated with greater opacity values under reflected light. On the contrary, slow growth period are associated with lower opacity values. From the inspection of the variations of the map of the relative opacities with respect to local shape curvatures, not all otolith zones undergo the same process.

[Figure 7 about here.]

The comparison of the evolution of the growth increments and of the associated opacities, as a function of the age, along four different growth axis chosen in the dorsal, ventral, distal and proximal zone, further illustrate these points (Fig.7). Whereas the variations of the opacity are synchronous for the four growth axis, this is not as clear for the growth increments. For instance, the growth along the ventral axis does not follow the evolution of the growths along the dorsal and distal axis between age 2 to 3 . Opacity and growth follow similar decreasing trends when the fish gets older in the four otolith zones. With a view to evaluating how similar or different the otolith growth is in the distal, proximal, dorsal and ventral zones, a factor analysis has been carried using the five local features. This factor analysis (FA) (Fig.8) shows that the different otolith zones are clearly separated in the FA feature space (t-test, $p<0.001$ ). The factor space is mainly structured by curvature and growth features, whereas the contribution of opacity characteristics is weaker. Not only the mean characteristics appear different between the different otolith zones, but also the relations between these features. A similar analysis has been carried out for the nine other otolith sections which confirm this result (t-tests, $p<0.001$ in all cases). This Factor analysis also indicates that positive correlations may be analyzed especially, between ring curvature, growth increment and opacity features. 
A correlation analysis is detailed for the reference pollock otolith section (Fig.9). Regarding growth

features, positive and meaningful correlations $(p<0.001)$ are found between ring curvature and growth increment as well as between ring curvature and growth anisotropy. Concerning opacity features, significant correlations are observed between growth increment and opacity, as well as between growth anisotropy and relative opacity. Note that no significant correlation is retrieved between ring curvature and opacity.

[Figure 9 about here.]

These correlations are evaluated over the whole section. However, as indicated by the factor analysis, the different otolith zones may not exhibit the same type of relations. A similar correlation analysis is then also carried out for the dorsal, distal, ventral and proximal otolith zones. Results are reported for the considered set of ten transverse pollock otolith sections (Fig.10). Growth increment and ring curvature are significantly correlated to ring curvature in the ventral and dorsal zones as well as globally for all sections. In the distal and proximal zones, growth and ring curvature are weakly correlated, and growth anisotropy and ring curvature are negatively correlated. These negative correlations are significant for only four sections over ten in the distal zones. It should be stressed that the distal and proximal zones are the ones in which negative curvature values are found, i.e. areas in which the shape is not locally convex with respect to the otolith core.

Regarding opacity features, the global correlations are significant for only three sections over ten between opacity features and ring curvature. Greater correlations are observed between respectively growth and opacity, and, growth anisotropy and relative opacity. They are significant for nine sections over ten. Focusing on the different otolith zones, the dorsal zone depict similar characteristics. In the ventral zone, opacity is significantly correlated to growth for all sections, but the correlation between relative opacity and growth anisotropy is significant for only six sections. The distal and proximal zones do not follow this 
pattern. In the distal zone, growth and opacity are mostly significantly correlated (nine over ten sections), but both negative and positive correlations are observed between relative opacity and growth anisotropy, only very few being significant. In the proximal zone, opposite observations can be made: relative opacity and growth anisotropy are positively and significantly correlated, but growth and opacity are positively and significantly correlated for only six sections, one section depicting even a significant negative correlation.

[Figure 10 about here.]

\section{Discussion}

Model genericity and contributions w.r.t. previous work A new tool has been presented for the reconstruction of individual shape histories of otolith sections. Relying on the representation of the accretionary growth of fish otoliths by a potential function whose level-lines are the successive 2D shapes of the otolith in a given observation plane, the proposed variational formulation exploits orientation-related cues to fit this model to a given otolith image. Compared to the previous work presented in [Alvarez et al., 2007], new contributions are brought:

- Contrary to [Alvarez et al., 2007], we do not only rely on shape interpolation between internal ring constraints set manually. The image content, more precisely the estimated field of local image orientations, is exploited to constrain the estimation of the model. This flexibility permits an automated reconstruction of the otolith shape history using only the position of the nucleus as an internal constraint. The process can be improved using additional internal ring constraints, set either as closed ring or open curves.

- In [Alvarez et al., 2007] a parametric model is proposed. The underlying assumption is that model parameters are constant along radials from the core to the edge. More precisely, the local growth 
magnitude is modeled as a linear function of the ring curvature. The analysis of the 2D otolith growth carried out in this study for transverse sections of pollock otoliths shows that this empirical assumption is not satisfied for the processed example. In contrast, we propose a non-parametric and generic model. This non-parametric setting is proven robust and flexible to account both for elliptic-like samples, such as plaice otoliths, and for more complex shapes, such as those of pollock and cod otoliths. Being non-parametric, our approach also provides the mean for carrying out the analysis of the 2D otolith growth with no particular assumption that may constrain and limit this analysis.

- Our model also distinguishes the geometric component of the otolith shape history and the associated growth pattern. While the image-based variational minimization solves for the first task, additional information set manually such as the positions of the annual rings permits calibrating the level-set shape model using the fish age as an actual time scale.

- Appropriate minimization methods have been developed so that the computational time required for model fitting is typically of a minute for a 1000x1000 image of an otolith section. This processing time includes both the computation of the orientation field and the estimation of the potential $U$ given this orientation field. Compared to the high computational load required by the scheme proposed in [Alvarez et al., 2007] (several hours for a 1000x1000 image), it provides the mean for exploiting the estimated level-set representation for various tasks and applications.

Given the genericity of the propose framework, the application to other examples of accretionary growth process, such as shellfish or corals [Ubutaka, 2003], will be considered. An other issue of interest in future work would be the reconstruction and the analysis of the dynamics of the 3D shape of the otolith. Such 3D representations could be deduced from multiple parallel 2D otolith sections as used for 3D brain mapping from 2D scanned slices and would mainly require specific technical developments to acquire such images in 
successive otolith plane.

New local otolith signatures and otolith growth analysis Regarding otolith growth analysis, we have proposed new local quantitative features of 2D otolith growth, i.e. ring curvature, growth increment, growth anisotropy and relative opacity, which are by-products of the estimation level-set representation. There are viewed as new means for locally characterizing accretion process.

Initial results are reported in this study from the analysis of a set of ten transverse sections of pollock otoliths. Regarding 2D otolith growth, reported results stress how asynchronous the growth along different growth axis can be and emphasize the need for advanced 2D (and possibly 3D) tools for growth analysis. The quantitative statistical analysis exhibits significant positive correlation between otolith opacity and growth, and, relative opacity and growth anisotropy. This can be regarded as a quantitative evaluation of the strength of these relationships which are broadly known and evaluated at a global level [Panfili et al., 2002, Campana and Thorrold, 2001, Hussy and Mosegaard, 2004]. Focusing on the latter relationship, the absence of a significant correlation between relative opacity and curvature points out that local opacity variations are not constrained by shape characteristics. It suggests that local otolith growth and opacity can be viewed as a modulation of two factors: a global factor related to fish metabolism and environment and a local factor related to the local physico-chemical characteristics of the endolymph [Allemand et al., 2007]. Besides, the quantitative characterization of these relationships in the different otolith zones (i.e., ventral, dorsal, proximal and dorsal zones) indicates that for some relationships (e.g., relative opacity vs. growth anisotropy) a global mean law may be relevant. In contrast, for other relationships (e.g., curvature vs. local growth), zone-dependent relationships seem more appropriate.

In future work the proposed quantitative 2D analysis framework will be exploited to further investigate at the individual level the relationships between physico-chemical otolith features and otolith growth and opacity, as well as the relationships between otolith opacity, otolith growth, endolymph heterogeneity 
[Allemand et al., 2007, Payan et al., 1999], fish metabolism and environmental variables (e.g., temperature and salinity).

A generic tool for otolith analysis and applications The proposed approach performs the extraction of the series of the successive otolith shapes. Otolith shape has been proven to be among the relevant features for species and/or stock discrimination issues [Campana and Casselman, 1993, de Pontual and Prouzet, 1987, Parisi-Barabad et al., 2005]. Such application generally relies on the characterization of the otolith outline, for instance by Fourier descriptors. Considering the whole and/or subsequences of the individual shape histories considerably enriches the available characterization, as it intrinsically conveys both shape and growth information. Curvature and growth anisotropy maps may also be of interest for these issues. These novel shape-based features should lead to signficant improvements of stock and species discrimination from fish otoliths.

From the proposed otolith growth representation, an adapted polar-like coordinate system, where the angular information $\theta$ refers to a growth axis (indexed w.r.t. a point along the outline) and the radius information $\rho$ to an age, has been proposed for analyzing 2D otolith sections. Exemplified in our study for the spatial and temporal analysis of otolith growth features, this otolith-specific coordinate system is of broad interest: for instance, for standardizing the analysis of one-dimensional transects for one or several individuals or evaluating differences or similarities w.r.t. otolith sampling zones. Fish length backcalculation from otolith measurements [Campana and Thorrold, 2001, Panfili et al., 2002] is another application. Whereas backcalcultation laws typically exploits only one specific reference axis, the proposed setting provides the mean for extending such laws to any growth axis as well as ensuring the standardization of the reference growth axis (Fig.7). Regarding the extraction of chemical signatures, the proposed framework can contribute to the standardization and the automatic programming of transect characteristics (e.g., spots locations), for instance for the analysis of trace elements or isotopes by using WDS, LA-ICPMS or SIMS 


\footnotetext{
${ }^{2} \mathrm{An}$ animated version can be visualized at public.enst-bretagne.eu/ rfablet/mottolith.htm
} image and oxygen isotope signatures of a hake otolith (Desenfant et al unpublished) ${ }^{2}$.

[Figure 11 about here.]

[de Pontual and Geffen, 2003]. In some applications, microdrilling is required for the subsequent analyses of isotopic concentrations by using IRMS, MC-ICPMS or TIMS [Hoie et al., 2004, Klaue et al., 2002, Alvarez et al., 2005]. The definition of the micro-drilling trajectories is of primary importance to ensure that growth-consistent otolith zones are sampled. Microdrilling trajectories are defined by both a prior manual recording of otolith reference lines and an interpolation between those lines. This quite tedious process presents a risk of mismatching intermediate trajectories and internal otolith rings, resulting in noisy chemical measurements. It has been recognized that such analytical issues might be a limiting factor for a joint analysis of otolith $\delta^{18} \mathrm{O}$ (a proxy of water temperature) and otolith opacity [Hoie et al., 2004]. In contrast, from the proposed framework, micro-drilling trajectories could be defined from portions of the estimated level-set representation of the otolith growth. In the same context of otolith microchemistry, the proposed representation also provides new means for performing a joint analysis and a fusion between multiple chemical signatures, as well as with image-based otolith features (e.g., opacity), issuesbeing far from trivial. An illustration of the potential of the proposed framework is reported (Fig.11) for the fusion of the opacity

Another important application is the modelling of th formation of fish otoliths. The contributions are two-fold. The proposed approach first permits investigating, at the individual level, a quantitative characterization of 2D otolith growth and determining the relevant relationship between otolith features. Previous work [Hussy and Mosegaard, 2004] relied on global characteristics (e.g., mean otolith opacity vs. mean otolith growth). Such a global analysis is rather coarse to formulate and test modelling hypothesis. In contrast, such issues can be dealt with the proposed scheme. For instance, hypothesis on otolith growth considered in [Alvarez et al., 2007] (e.g., that local growth can be radially parameterized) are shown not 
to be satisfied and reported results suggest that an exponential model might be appropriate to relate local growth anisotropy and relative opacity. Similarly, the analysis of temporal shifts in the accretion regimes (e.g., checks, seasonal opacity changes,...) will be easier at an individual level given inter-individual otolith variabilities. The second contribution resorts to the extension of one-dimensional otolith models as proposed in [Hussy et al., 2004] to a joint 2D growth-opacity predictive model of the accretion of the otolith ${ }^{3}$. Such a model would be of great interest to better understand the conditions of the formation of the successive opaque and translucent layers of the biomineral.

\section{Acknowledgements}

We are grateful to Andre Ogor for the acquisition of the otolith images. We also thank F. Cao for fruitful discussion on the minimization of variational issue based on level-set representations. This work has been carried out with the financial support from the Commission of the European Communities, specific program "Specific Support to Policies", SSP8-044132 "AFISA". It does not necessarily reflect its views and in no way anticipates the Commission's future policy in this area. This work has also been supported by national ANR grant "OTOCAL".

\section{References}

[Allemand et al., 2007] Allemand, D., Mayer-Gostan, N., de Pontual, H., Boeuf, G., and Payan, P. (2007). Handbook of biomineralization, chapter Fish otolith calcification in relation to endolymph chemistry, pages 291-308. Ed. E. Bauerlein, Wiley.

${ }^{3}$ An example of a numerical simulation of an otolith growth model can be visualized at public.enstbretagne.eu/ rfablet/mottolith.htm 
[Alvarez et al., 2005] Alvarez, A., Palmer, M., Tomas, J., and Morales-Nin, B. (2005). Validation of sr isotopes in otoliths by laser ablation multicollector inductively coupled plasma mass spectrometry (lamc-icpms): opening avenues in fisheries science applications. Can. Jal Fish. Aq. Sc., 62(11):2425-2430.

[Alvarez et al., 2007] Alvarez, A., Palmer, M., Tomas, J., and Morales-Nin, B. (2007). A two-dimensionnal otolith growth inverse model. Jal of Fish Biology. In Press.

[Campana and Casselman, 1993] Campana, S. and Casselman, J. (1993). Stock discrimination using otolith shape analysis. Can. Jal of Fish. Res. and Aqu. Sc., 50:1062-1083.

[Campana and Thorrold, 2001] Campana, S. and Thorrold, S. (2001). Otoliths, increments, and elements: keys to a comprehensive understanding of fish populations? Can. Jal of Fish. Res. and Aqu. Sc., 58(1):3038.

[Cao and Fablet, 2006] Cao, F. and Fablet, R. (2006). Automatic morphological detection of otolith nucleus. Pattern Recognition Letters, 27(6):658-666.

[Chessel et al., 2006] Chessel, A., Cao, F., and Fablet, R. (2006). Interpolation of orientation: an axiomatic approach. In Proc. of European Conference on Computer Vision, ECCV06, LNCS 3954, pages 241-254, Graz.

[Cohen, 2005] Cohen, L. D. (2005). Minimal paths and fast marching methods for image analysis. In Paragios, N., Chen, Y., and Faugeras, O., editors, Mathematical Models in Computer Vision: The Handbook. Springer.

[de Pontual and Geffen, 2003] de Pontual, H. and Geffen, A. (2003). Otolith microchemistry. In J. Panfili, H. De Pontual, H. T. and Wright, P., editors, Manual of Fish Sclerochronology, pages 243-304. Editions Ifremer. 
[de Pontual and Prouzet, 1987] de Pontual, H. and Prouzet, P. (1987). Atlantic salmon, Salmo salar 1., stock discrimination by scale-shape analysis. Aquaculture and Fisheries Management, 18:277-289.

[de Pontual and Prouzet, 1988] de Pontual, H. and Prouzet, P. (1988). Numerical analysis of scale morphology to discriminate between atlantic salmon stocks. Aqu. Liv. Res., 1(1):17-27.

[Desolneux et al., 2001] Desolneux, A., Moisan, L., and Morel, J. (2001). Edge detection by helmoltz principle. Journal of Mathematical Imaging and Vision, 14(3):271-284.

[Desolneux et al., 2003] Desolneux, A., Moisan, L., and Morel, J.-M. (2003). A grouping principle and four applications. PAMI, 25(4):508-513.

[Fablet, 2006] Fablet, R. (2006). Semi-local extraction of ring structures in images of biological hard tissues: application to the bayesian interpretation of fish otoliths for age and growth estimation. Canadian Journal on Fisheries and Aquatic Sciences, 43(6):1414-1428.

[Fablet et al., 2008a] Fablet, R., Chessel, A., Carbini, S., Benzinou, A., and de Pontual, H. (2008a). Mottolith: a new image-based tool for otolith growth analysis and modeling. Technical report, Ifremer/LASAA, http://perso.enst-bretagne.fr/ronanfablet.

[Fablet et al., 2008b] Fablet, R., Chessel, S. P. A., Benzinou, A., and Cao, F. (2008b). 2D image-based reconstruction of shape deformation of biological structures using a variational level-set approach. Computer Vision and Image Understanding. To appear.

[Fablet et al., 2007] Fablet, R., Daverat, F., and de Pontual, H. (2007). Unsupervised bayesian reconstruction of individual life histories chronologies from otolith signatures: case study of Sr:Ca transects of eel (anguilla anguilla) otoliths. Can. Jal of Fish. Res. and Aqu. Sc., 64:152-165. 
[Guillaud et al., 2002] Guillaud, A., Benzinou, A., Troadec, H., Rodin, V., and Bihan, J. L. (2002). Autonomous agents for edge detection and continuity perception on otolith images. Im. and Vis. Comp., 20(13-14):955-968.

[Hoberman and Jensen, 1962] Hoberman, J. and Jensen, A. (1962). The growth rate of new england pollock. Trans. Am. Fish. Soc., 91:227-228.

[Hoie et al., 2008] Hoie, H., Folkvord, A., Mosegaard, H., Li, L., Clausen, L., Norberg, B., and Geffen, A. (2008). Restricted fish feeding reduces cod otolith opacity. Jal Appl. Icht., 0(0):xx-xx.

[Hoie et al., 2004] Hoie, H., Otterleib, E., and Folkvord, A. (2004). Temperature-dependent fractionation of stable oxygen isotopes in otoliths of juvenile cod (gadus morhua 1.). ICES Jal of Marine Science, 61(2):243-251.

[Hussy and Mosegaard, 2004] Hussy, K. and Mosegaard, H. (2004). Atlantic cod (gadus morhua) growth and otolith accretion characteristics modelled in a bioenergetics context. Can. Jal of Fish. Res. and Aqu. Sc., 61(6):1021-1031.

[Hussy et al., 2004] Hussy, K., Mosegaard, H., and Jessen, F. (2004). Effect of age and temperature on amino acid composition and the content of different protein types of juvenile atlantic cod (gadus morhua) otoliths. Can. Jal of Fish. Res. and Aqu. Sc., 61(6).

[Klaue et al., 2002] Klaue, A., Blum, J., Folt, C., Nislow, K., and Kennedy, B. (2002). Robust and efficient detection of salient convex groups. Can. Jal Fis. Aq. Sc., 59(6):925-929.

[Palmer et al., 2005] Palmer, M., Alvarez, A., Tomas, J., and Morales-Nin, B. (2005). A new method for robust feature extraction of otolith growth marks using fingerprint recognition methods. Marine and Freshwater Research, 56:791-794. 
[Panfili et al., 2002] Panfili, J., Pontual, H. D., Troadec, H., and Wright, P. (2002). Manual of Fish Sclerochronology. Editions Ifremer.

[Parisi-Barabad et al., 2005] Parisi-Barabad, V., Lombarte, A., Garcia-Ladona, E., Cabestany, J., Piera, J., and Chico, O. (2005). Otolith shape contour analysis using affine transformation invariant wavelet transforms and curvature scale space representation. Marine and Freshwater Research, 56(5):795-804.

[Payan et al., 1999] Payan, P., Edeyer, A., Pontual, H. D., Borelli, G., Boeuf, G., and Mayer-Gostan, N. (1999). Chemical composiion of saccular endolymph and otolith in fish inner ear: lack of spatial uniformity. Am. Jal Phys.: Reg. Int. Comp. Phys., 277(1):123-131.

[Traodec et al., 2000] Traodec, H., Benzinou, A., Rodin, V., and Bihan, J. L. (2000). Use of deformable templates for otolith 2D growth ring detection by digital image processing. Jal of Fish. Res., 46(1-3):155163.

[Ubutaka, 2003] Ubutaka, T. (2003). Pattern of growth rate around aperture and shell form in Bivalvia: a theoretical morphological study. Paleobiology, 29(4):480-491. 


\section{List of Figures}

1 Level-set representation of the accretionary growth of fish otoliths: each level-line of the increasing potential function represents the shape of the otolith at a given age. . . . . . . . 26

2 Reconstruction of the series of growth shapes for a pollock (Pollachius virens) otolith image depicted above: first row, original otolith image (left) and extracted orientation field using the AMLE (right); second row, estimated potential function represented as a 3D surface (left) and estimated shape history superimposed to the image (right). The constraints (here, the otolith center) are superimposed to the otolith image as black curves, and the equally sampled level sets of the reconstructed potential function $U$ as white curves. . . . . . . . . . . 27

3 Reconstruction of the individual shape histories for three fish otoliths: for a plaice (Pleuronectes platessa) otolith (first row), a cod (Gadhus morua) otolith (second row), and a whiting (Merlangius merlangus) otolith (third row. For each row, the otolith image and the series of shape superimposed to the otolith image are reported. Only the otolith center and the outline are used as constraints. . . . . . . . . . . . . . . . . . . . . . . . . . .

4 Examples of the automated extraction of ring structures in three otolith images: an image of pollock otolith section (first row), an image of a cod otolith section (second row), an image of a hake otolith section (third row). The otolith image with the curves detected using the proposed a contrario approach is reported (left), as well as the set of detected curves alone (center). A comparison to the results obtained from the template-based method (right) described in [Fablet, 2006] is also displayed. . . . . . . . . . . . . . . . . .

5 Growth axis extraction: original otolith images (left column), extracted growth axis superimposed to the otolith image (right column). The processed otolith images are the one reported in Fig. 2 and $3 . \ldots$. . . . . . . . . . . . . . . . . . . . . . . . . . .

6 Quantitative analysis of the 2D otolith growth: from left to right, and top to bottom, opacity acquired under reflected light of a 5-year pollock otolith section, otolith growth, ring curvature, otolith growth anisotropy, relative otolith opacity. . . . . . . . . . . . . . . . 31

7 Comparison of the features of the otolith growth along the main growth axis of the dorsal, distal, ventral and proximal zones: otolith image with superimposed growth axis (top), associated growth increment series (bottom left), associated opacity series (bottom right). . . 32

8 Projection of the otolith growth features in the frame of the two first factors of a factor analysis (FA): otolith image with superimposed growth axis corresponding to the four otolith zones considered for the factor analysis (i.e., the dorsal, distal, ventral and proximal zones of the otolith) (top), position of the different otolith growth features (growth increment, curvature, opacity, growth anisotropy, relative opacity) (black lines), data set associated with the dorsal, distal, ventral and proximal otolith zones (respectively, star, square, circle and cross markers) (bottom). . . . . . . . . . . . . . . . . . . . . . . .

9 Correlation analysis applied to the otolith growth features (growth, growth anisotropy, ring curvature, opacity and relative opacity) for the pollock otolith section depicted in Fig.6: from left to right and top to bottom, ring curvature vs. growth, ring curvature vs. growth anisotropy, ring curvature vs. opacity, ring curvature vs. opacity, growth vs. opacity and growth anisotropy vs. relative opacity. Otolith growth features from age 0.5 to 4 are considered. For each plot are given the Pearson correlation coefficient and the associated p-statistic. 34 
10 Correlation analysis applied to the otolith growth features (growth, growth anisotropy, ring curvature, opacity and relative opacity) for a set of ten pollock otolith sections: from left to right and top to bottom, ring curvature vs. growth, ring curvature vs. growth anisotropy, ring curvature vs. opacity, ring curvature vs. opacity, growth vs. opacity and growth anisotropy vs. relative opacity. For each plot the Pearson correlation coefficients are reported as black dots for the four otolith zones (dorsal, distal, ventral, proximal) and the whole section. Otolith growth features from age 0.5 to 4 are considered. When meaningful (p-statistic below 0.001), the associated correlation value is moreover marked by a square. .

11 Illustration of the image-based fusion of oxygen isotope signatures and otolith opacity for a hake otolith: acquired transect of the oxygen isotope ratios $\delta^{18} \mathrm{O}$ superimposed to the otolith image acquired under reflected light (top), fused image comprising in the upper part opacity information and the lower part the interpolated $\delta^{18}$ O values. . . . . . . . . . . . . 36 


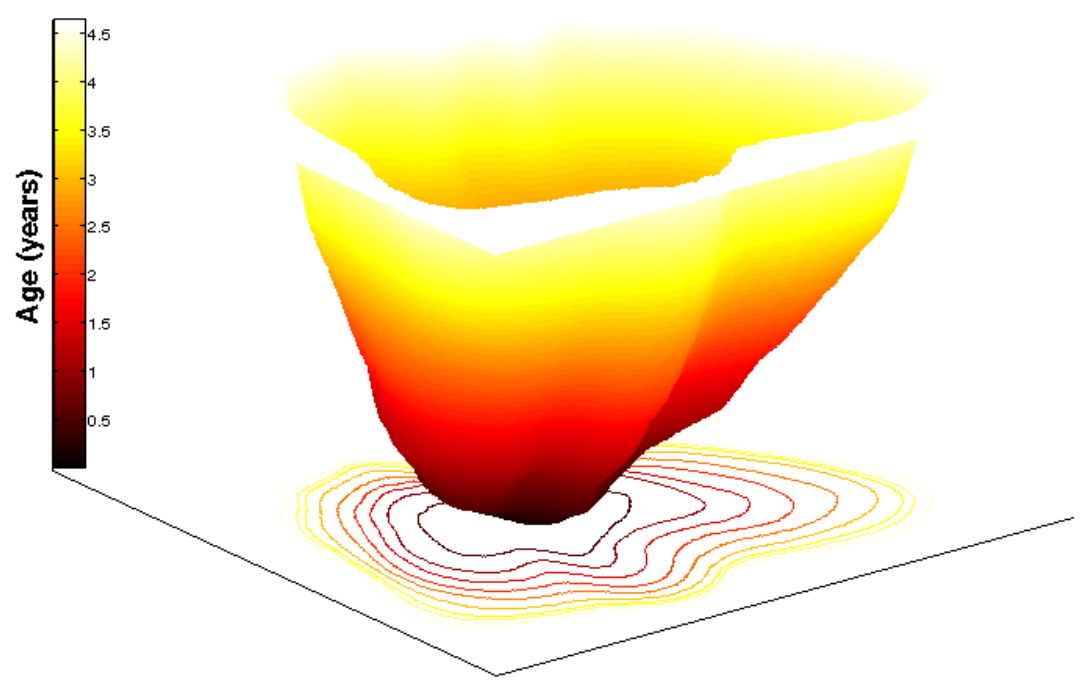

Figure 1: Level-set representation of the accretionary growth of fish otoliths: each level-line of the increasing potential function represents the shape of the otolith at a given age. 

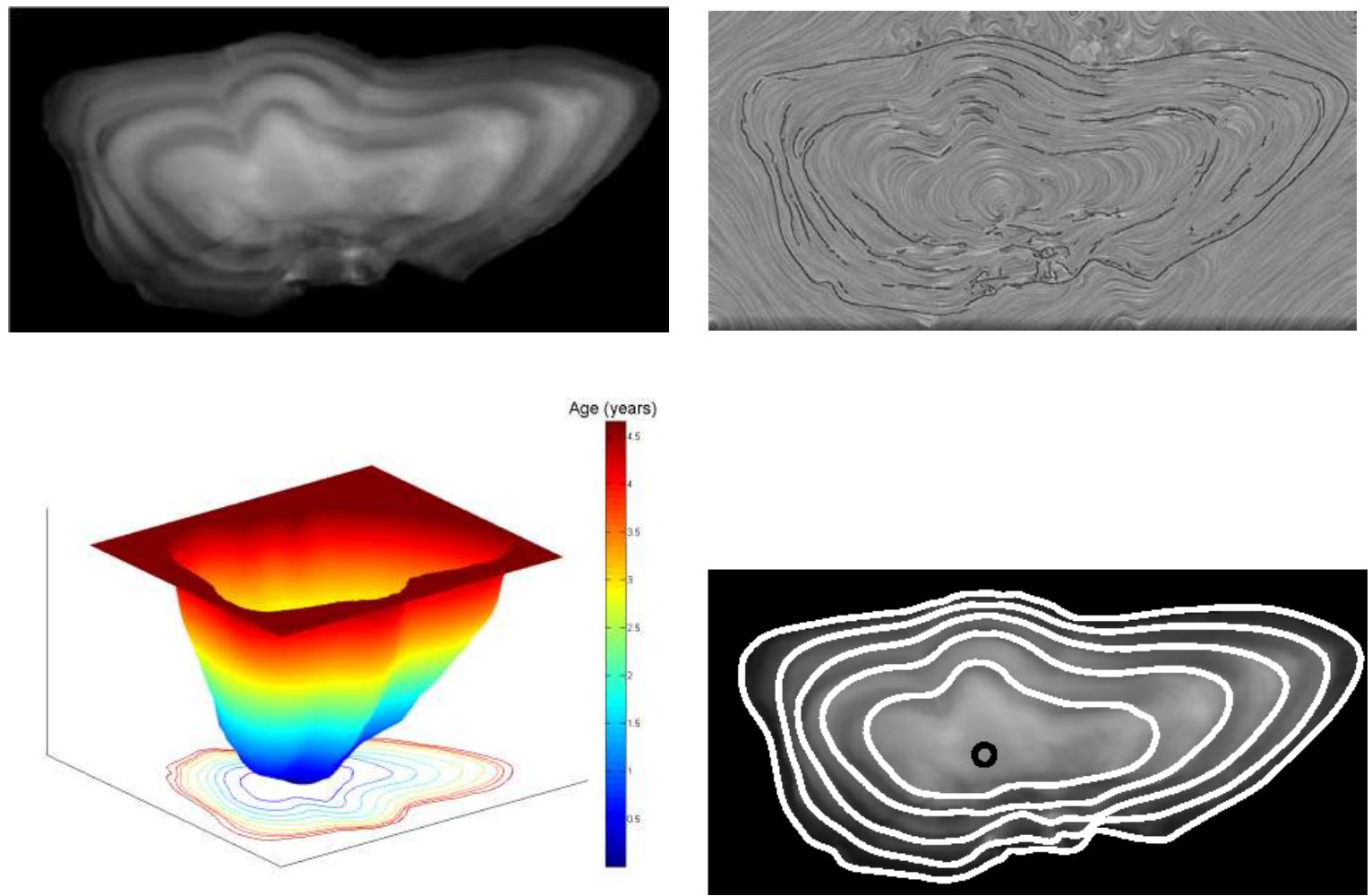

Figure 2: Reconstruction of the series of growth shapes for a pollock (Pollachius virens) otolith image depicted above: first row, original otolith image (left) and extracted orientation field using the AMLE (right); second row, estimated potential function represented as a 3D surface (left) and estimated shape history superimposed to the image (right). The constraints (here, the otolith center) are superimposed to the otolith image as black curves, and the equally sampled level sets of the reconstructed potential function $U$ as white curves. 

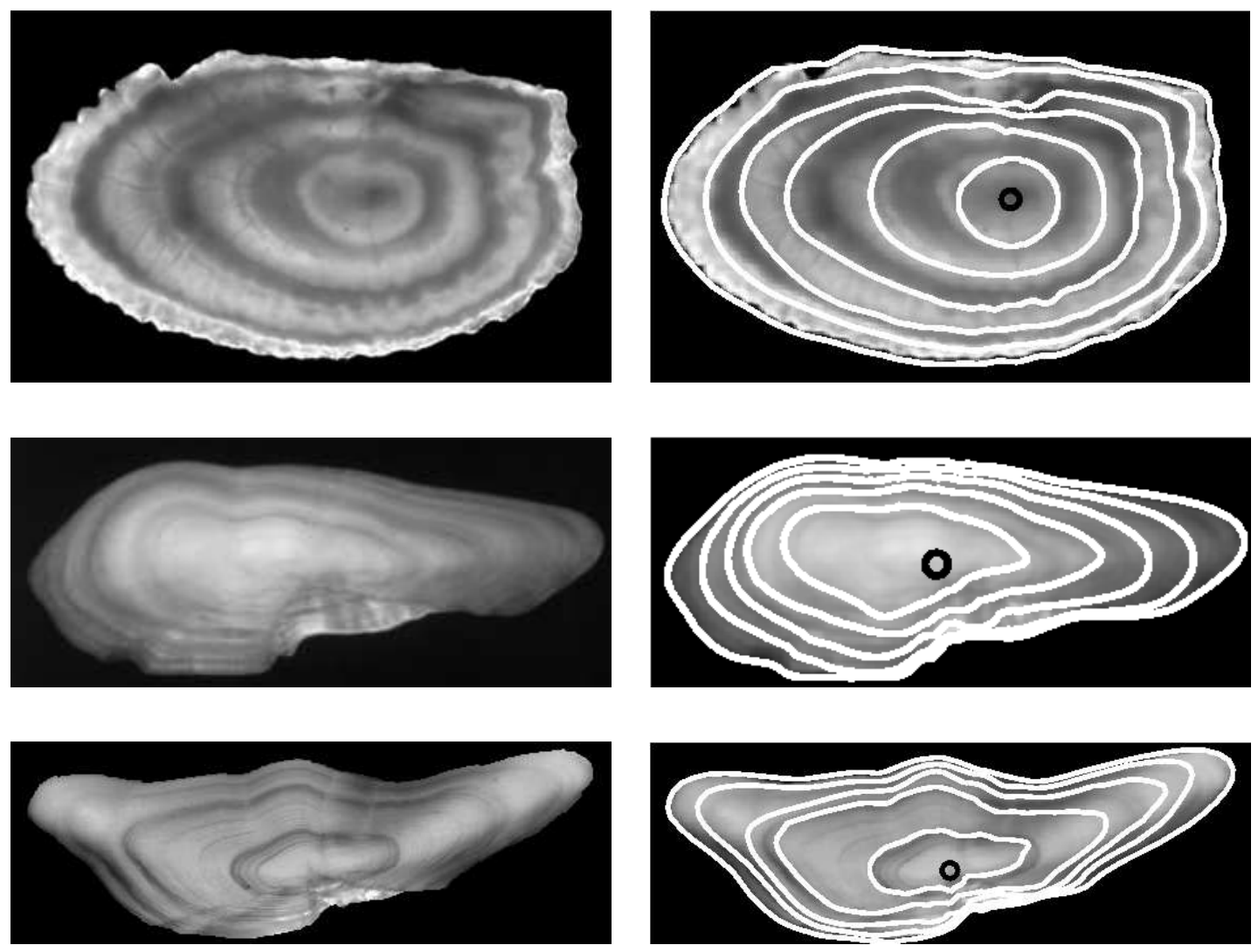

Figure 3: Reconstruction of the individual shape histories for three fish otoliths: for a plaice (Pleuronectes platessa) otolith (first row), a cod (Gadhus morua) otolith (second row), and a whiting (Merlangius merlangus) otolith (third row. For each row, the otolith image and the series of shape superimposed to the otolith image are reported. Only the otolith center and the outline are used as constraints. 
Proposed approach
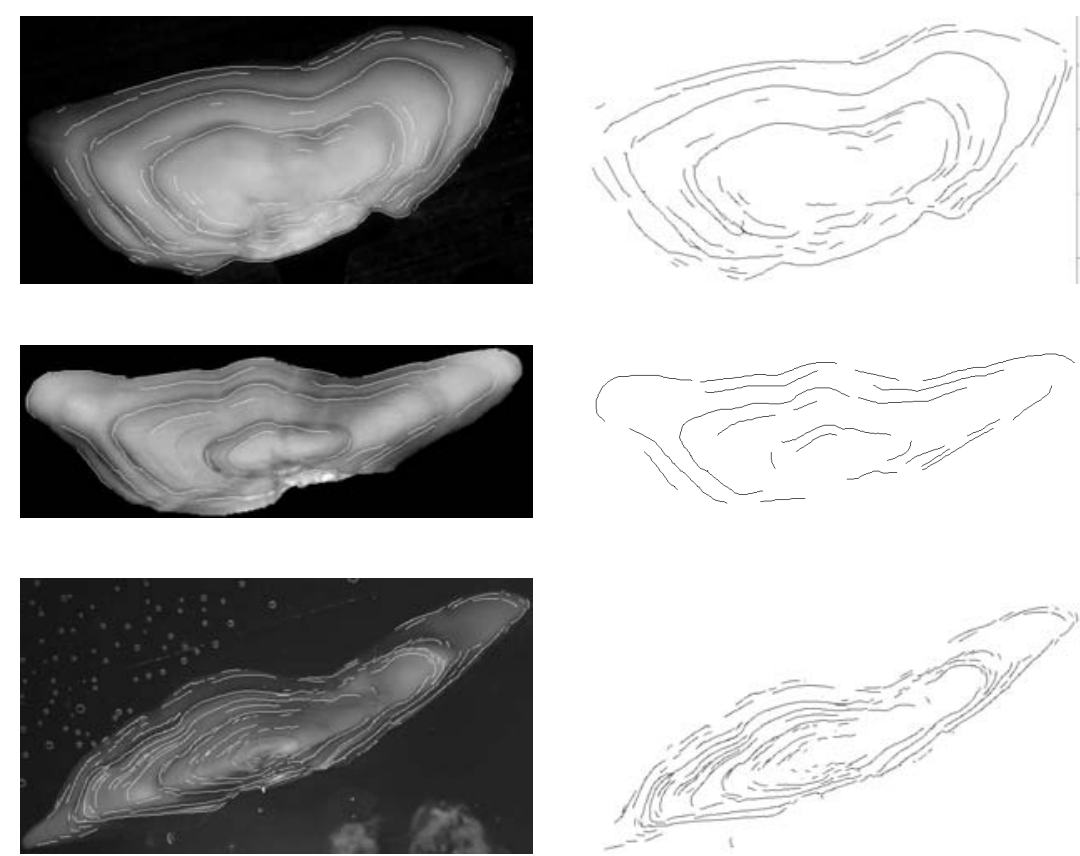

Method described in [Fablet, 2006]
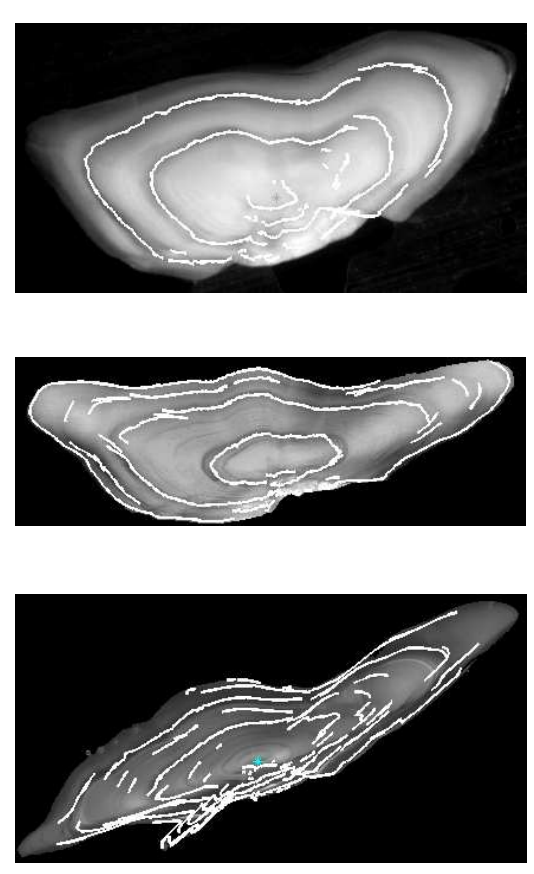

Figure 4: Examples of the automated extraction of ring structures in three otolith images: an image of pollock otolith section (first row), an image of a cod otolith section (second row), an image of a hake otolith section (third row). The otolith image with the curves detected using the proposed a contrario approach is reported (left), as well as the set of detected curves alone (center). A comparison to the results obtained from the template-based method (right) described in [Fablet, 2006] is also displayed. 

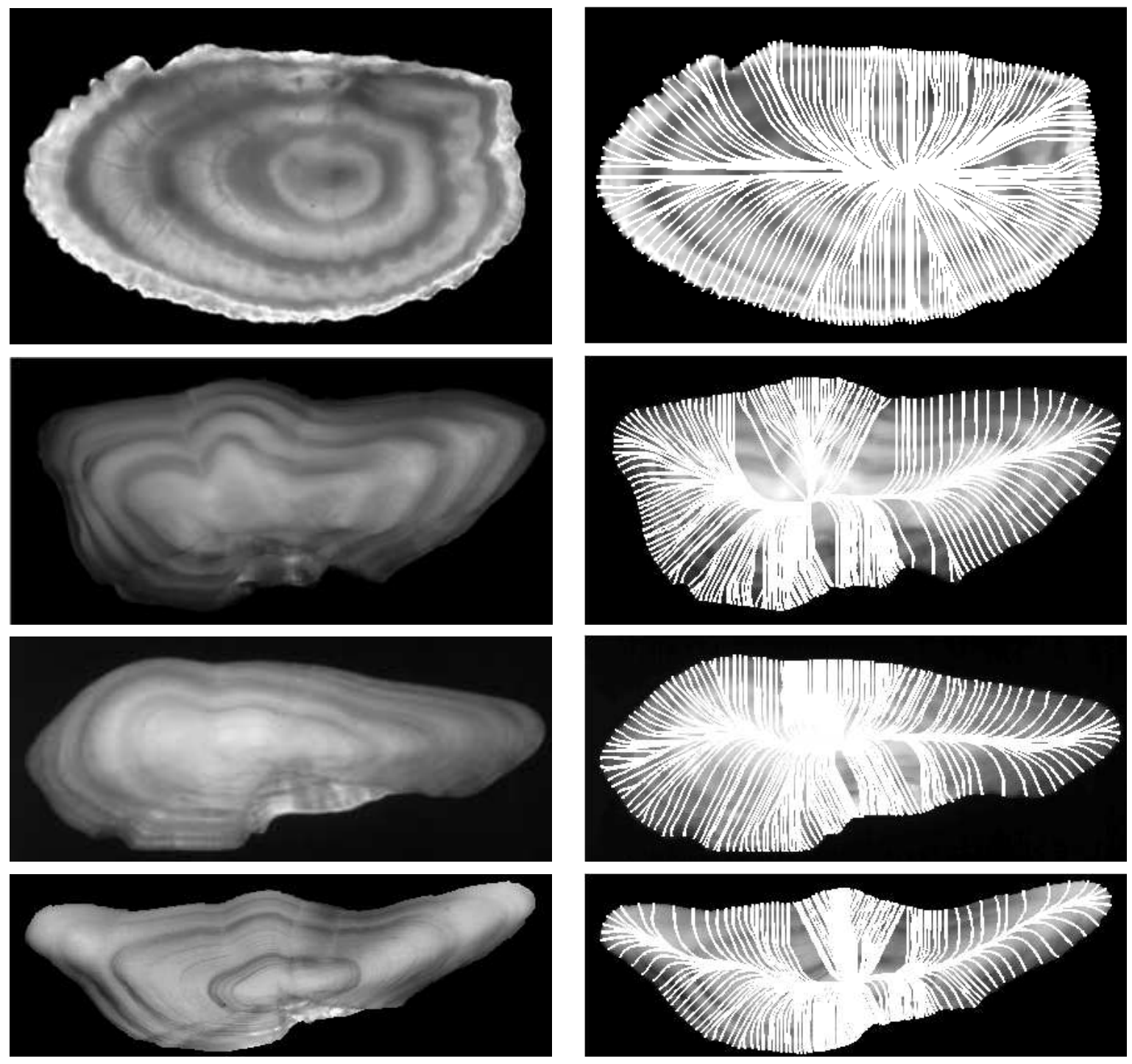

Figure 5: Growth axis extraction: original otolith images (left column), extracted growth axis superimposed to the otolith image (right column). The processed otolith images are the one reported in Fig. 2 and 3. 


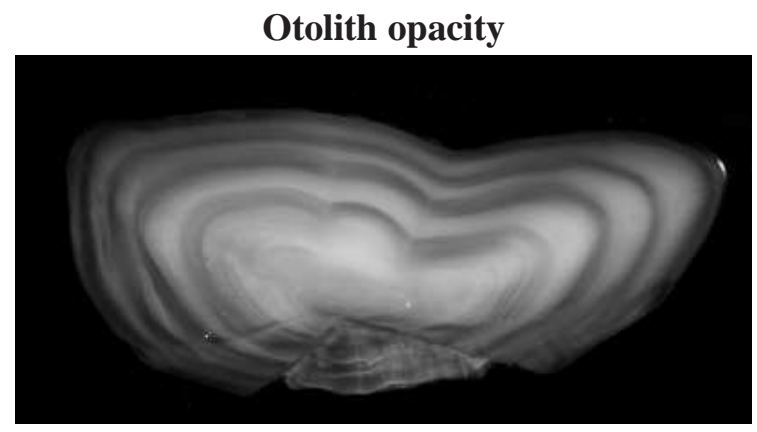

Otolith growth

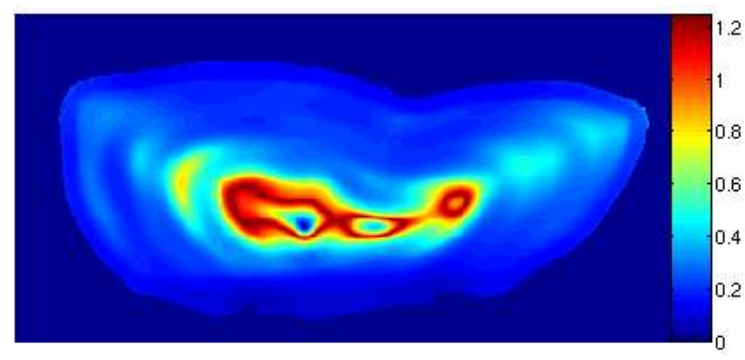

Otolith growth anisotropy

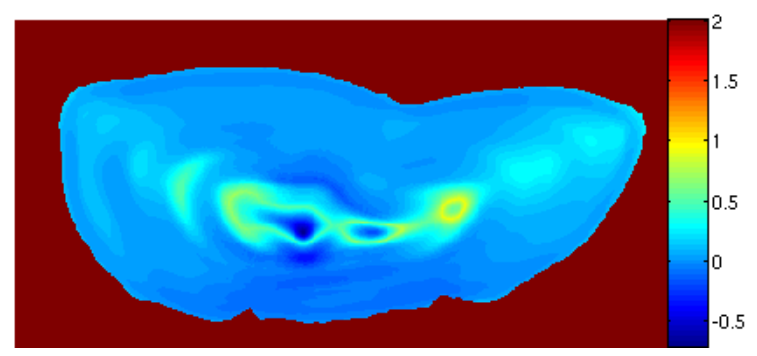

Ring curvature

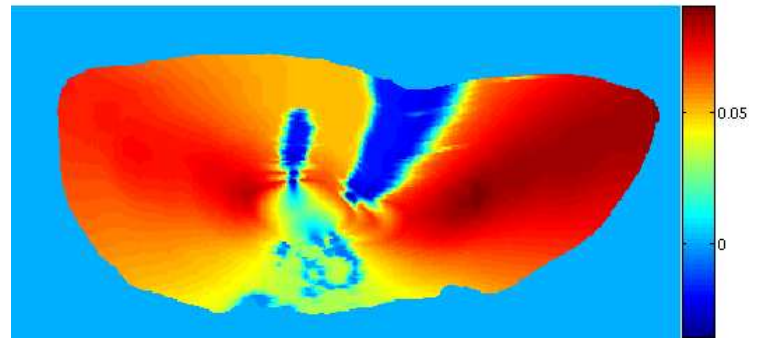

Relative otolith opacity

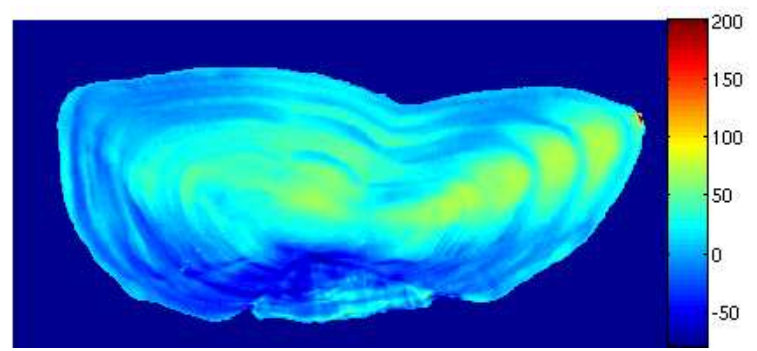

Figure 6: Quantitative analysis of the 2D otolith growth: from left to right, and top to bottom, opacity acquired under reflected light of a 5-year pollock otolith section, otolith growth, ring curvature, otolith growth anisotropy, relative otolith opacity. 

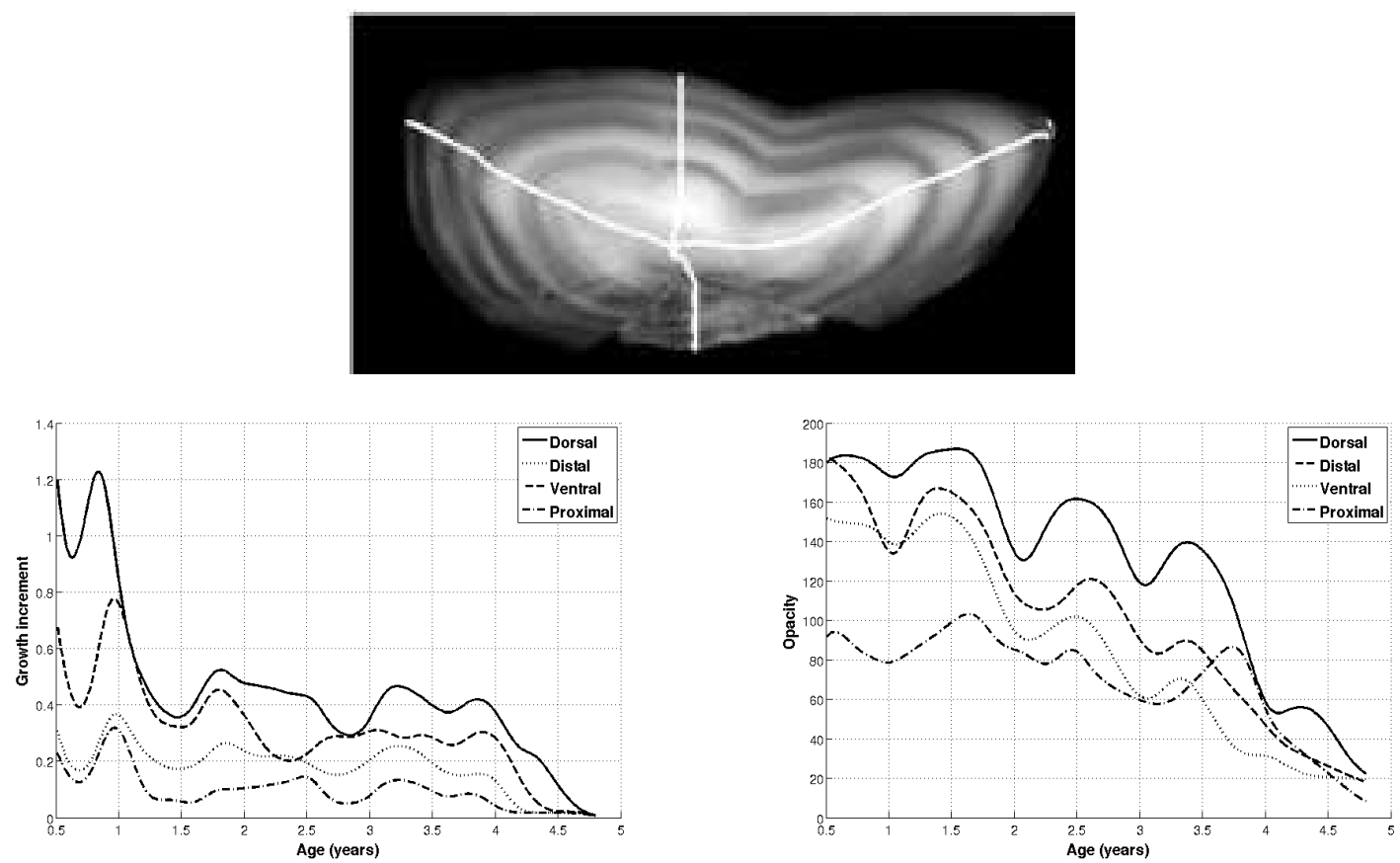

Figure 7: Comparison of the features of the otolith growth along the main growth axis of the dorsal, distal, ventral and proximal zones: otolith image with superimposed growth axis (top), associated growth increment series (bottom left), associated opacity series (bottom right). 

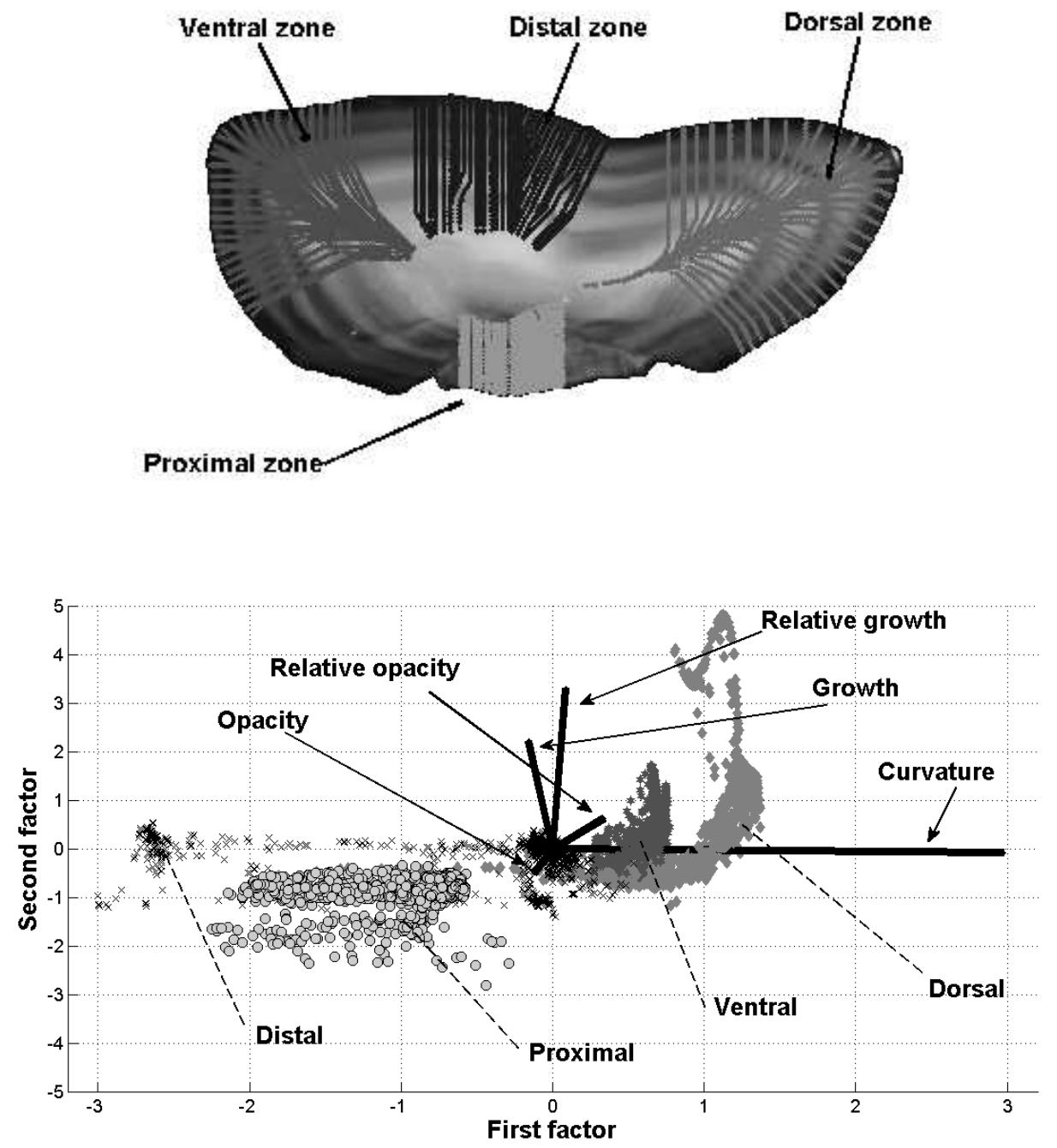

Figure 8: Projection of the otolith growth features in the frame of the two first factors of a factor analysis (FA): otolith image with superimposed growth axis corresponding to the four otolith zones considered for the factor analysis (i.e., the dorsal, distal, ventral and proximal zones of the otolith) (top), position of the different otolith growth features (growth increment, curvature, opacity, growth anisotropy, relative opacity) (black lines), data set associated with the dorsal, distal, ventral and proximal otolith zones (respectively, star, square, circle and cross markers) (bottom). 

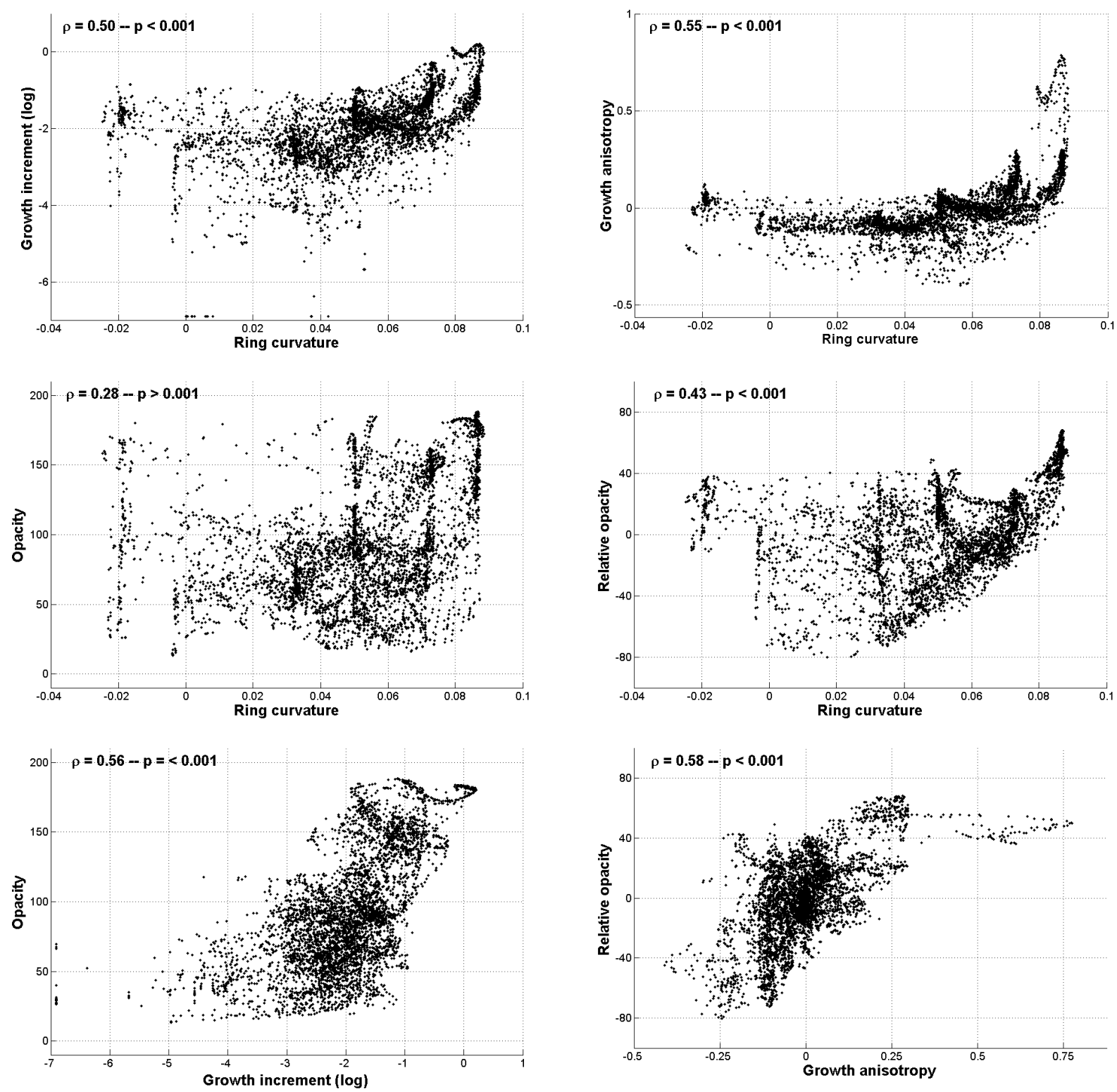

Figure 9: Correlation analysis applied to the otolith growth features (growth, growth anisotropy, ring curvature, opacity and relative opacity) for the pollock otolith section depicted in Fig.6: from left to right and top to bottom, ring curvature vs. growth, ring curvature vs. growth anisotropy, ring curvature vs. opacity, ring curvature vs. opacity, growth vs. opacity and growth anisotropy vs. relative opacity. Otolith growth features from age 0.5 to 4 are considered. For each plot are given the Pearson correlation coefficient and the associated p-statistic. 
Ring curvature vs. growth
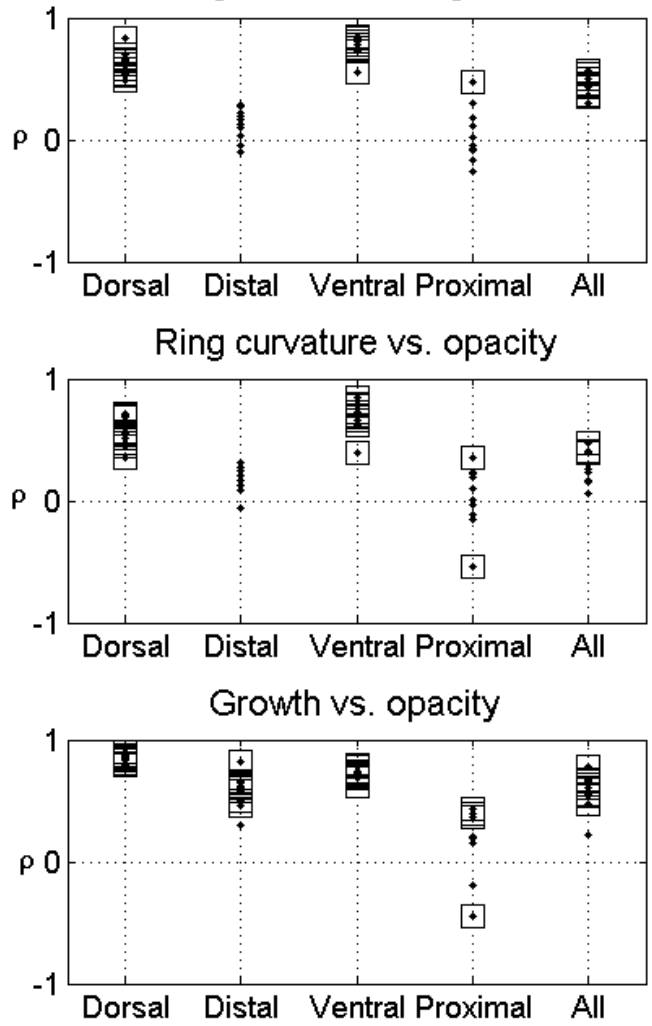

Ring curvature vs. growth anisotropy

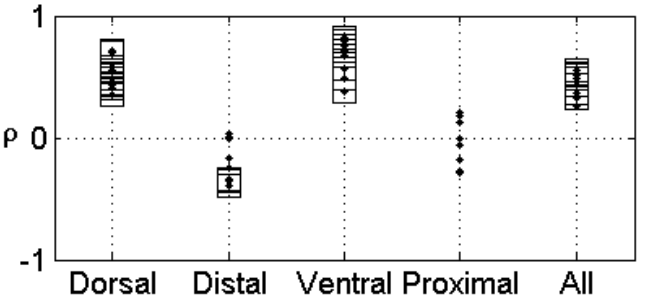

Ring curvature vs. relative opacity

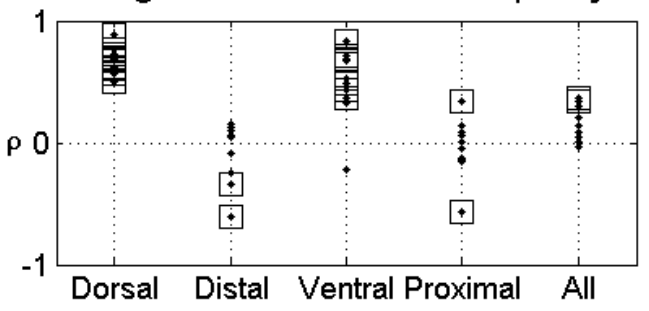

Growth anisotropy vs. relative opacity

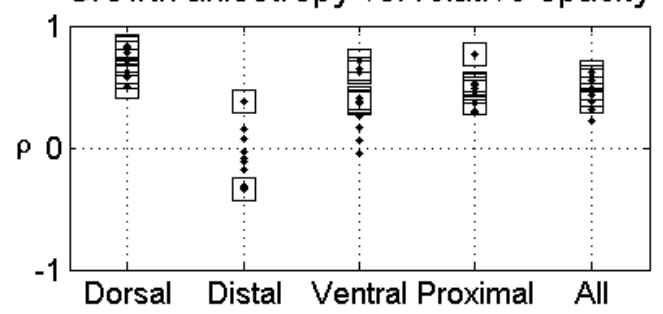

Figure 10: Correlation analysis applied to the otolith growth features (growth, growth anisotropy, ring curvature, opacity and relative opacity) for a set of ten pollock otolith sections: from left to right and top to bottom, ring curvature vs. growth, ring curvature vs. growth anisotropy, ring curvature vs. opacity, ring curvature vs. opacity, growth vs. opacity and growth anisotropy vs. relative opacity. For each plot the Pearson correlation coefficients are reported as black dots for the four otolith zones (dorsal, distal, ventral, proximal) and the whole section. Otolith growth features from age 0.5 to 4 are considered. When meaningful ( $p$-statistic below 0.001), the associated correlation value is moreover marked by a square. 


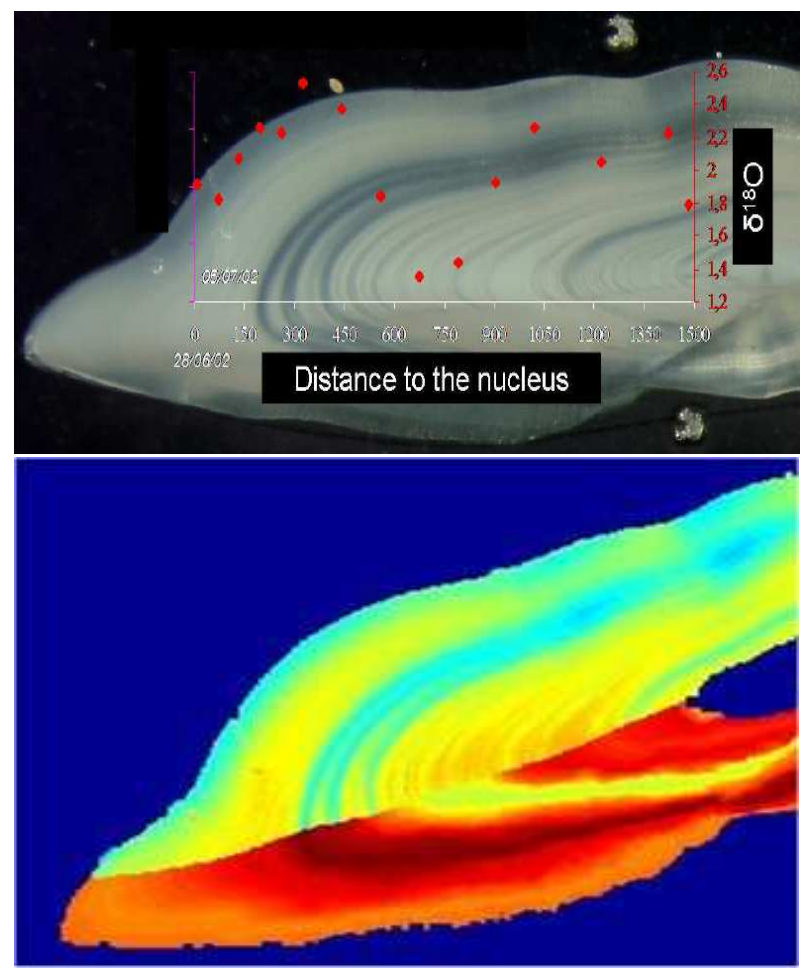

Figure 11: Illustration of the image-based fusion of oxygen isotope signatures and otolith opacity for a hake otolith: acquired transect of the oxygen isotope ratios $\delta^{18} O$ superimposed to the otolith image acquired under reflected light (top), fused image comprising in the upper part opacity information and the lower part the interpolated $\delta^{18} O$ values. 